\title{
An Analysis of the Causes of Job Burnout Among Flight Attendants
}

\author{
Pei Li \\ School of Tourism, Northwest Normal University, Anning District, Lanzhou City, Gansu Province, China \\ peili919@163.com
}

\begin{abstract}
With the advancement of society and the quickening pace of life, more and more employees are suffering from burnout. Burnout has a negative effect on an individual's performance in his workplace, his life outside of work and his health. And also, it could affect the performance of an organization. Hence, it is of great academic value and realistic significance to explore what causes job burnout. This paper focuses on flight attendants and analyzes the causes of burnout among them from the perspectives of career choices, working hours, working environment and organizational factors based on presenting their common characteristics and status of work.
\end{abstract} Keywords: flight attendants, job burnout, job planning, working hours, working environment, organizational factors

\section{空乘人员职业倦急的成因分析}

\begin{abstract}
裴莉
西北师范大学旅游学院, 安宁区, 兰州市, 甘肃省, 中国 peili919@163.com

\section{摘要}

随着社会文明的进步、生活节奏的加快，从业者出现职业倦急的现象日益突出，长期的职业倦急感不 仅给个体工作、生活、健康带来了严重危害, 而且也影响了整个组织的绩效。因此, 探究职业倦急的 形成机理具有重要的研究价值和现实意义。本文以空乘人员为研究对象, 在描述和呈现这一群体的共 性特征和工作现状的基础上，从职业选择的理性与非理性、非标准工作时间、特殊的工作环境及组织 因素等视角和维度分析了空乘人员职业倦急的成因。
\end{abstract}

关键词：空乘人员 职业倦怠 职业规划工作时间 工作环境 组织因素

\section{1. 问题的提出}

\section{1. 职业倦念概念的缘起和观点的呈现}

职业倦怠这一概念最早由美国临床心理学家费登 伯格 (Freudenberger) 于 1974 年提出, 他将情感枯竭和 动力丧失称为倦怠。[1]费登伯格认为在具有 “助人”性质 的行业中个体最容易出现身心疲劳, 即生理和心理的耗 竭状态, 当这类从业者将个体资源耗尽而无补充时, 就 会引发倦怠。对职业倦怠这一现象, 早期的调研对象主 要集中在公共服务及医疗保健领域的从业者身上, 比如 教师、医护人员、警察等, 美国社会心理学家马斯拉奇 (Maslach)就工作压力对这些从业者进行了采访, 进一步 提出了职业倦急的概念一一个体对长期压力下的工作
情绪及人际应激源作出反应而产生的心理综合症。[2]职 业倦怠常常表现为个体情感、态度以及行为上的衰竭状 态，如丧失对工作的热情、工作满意度下降、对工作意 义和价值的评价下降、持续出现无力感和消极情绪等。 在二十世纪八十年代, 倦急的评估和测量成为研究重点, 由此出现了许多不同的测量方法, 其中最为广泛使用的 方法是马斯拉奇和杰克逊(Jackson)开发的马斯拉奇职 业枯竭量表 (MBI)。[3]该量表包含三个子维度, 即情绪 耗竭、低成就感和人格解体, 且这三种维度的出现是一 个循序渐进的过程。随后还出现了一些关于职业倦怠区 分效度的研究, 如贝克 (Bakker) 等人通过职业枯竭量 表及其他测量方法说明了职业倦怠和抑郁症之间的区 别, ${ }^{[4]}$ 又如马斯拉奇和莱特 (Leiter) 解释了工作满意度 和职业倦怠之间存在负相关的关系。[5]

事实上，大众对职业倦怠也有许多不同的理解和看 法。有观点认为，理想主义者更容易出现职业倦急，因 为敬业者为实现自己的理想而努力工作，当他们的努力 


\section{2. 职业规划的理性和非理性选择与空乘人员 职业倦急感}

许多空乘从业人员选择这一职业并非经过理性的 思考和清晰的职业规划。首先, 在就业市场上, 职业的 可进入性差异很大，教育层次和技能要求是限制进入的 主要因素。航空公司对应聘者的主要评估标准是通过面 试感知应聘者的身体条件和人格特征, 即感知吸引力, 而对于空乘人员教育水平的要求相对较低, 目前各航空 公司的的最低学历要求是大专, 所以空乘职业的进入门 槛相对较低。其次，在目前竞争激烈的就业市场，相对 于其他服务行业, 空乘有着较高的社会地位和薪酬以及 看似优雅的工作环境, 这对于应聘者颇具诱惑力。再次, 在职业选择的过程中, 很多人选择成为空乘的原因是为 了满足自身旅行的愿望和期望改变单调的生活方式, 空 乘这一职业的某些属性契合了年轻人渴望新奇和追求 刺激的心理特征。但是工作带来的新鲜感是很短暂的, 当繁杂、重复的服务工作成为飞行的日常，工作热情很 快就会被职业倦怠所取代。第四, 性别角色的定位和女 性思维的束缚。在社会角色中, 女性是家庭生活的主要 维系者和日常家务的主要承担者。从前文对空乘人员工 作现状的描述中我们获悉, 空乘人员中女性占极高比例, 但很多年轻女性迫于现实因素把当空姐并未作为一个 为之奋斗的终生抱负, 只是作为找到稳定安逸工作或是 成为全职主妇之前的一个人生过渡阶段, 这使得她们不 愿在工作中投入巨大的热情和精力, 因此也很难从工作 中获得成就感。

\section{3. 非标准工作时间与空乘人员职业倦急感}

空乘人员工作时间的特殊性对其健康状况及家庭 生活带来的影响也是空乘人员产生职业倦急感的主要 因素。空乘人员的薪酬是按照飞行时数计算的, 夜间飞 行和跨时区飞行是不可避免的，不规律的作息时间、时 差反应及饮食的不规律对空乘人员的身心健康构成了 潜在的风险。有关研究表明, 空乘人员普遍存在生理节 律紊乱、慢性瘦劳以及长期失眠等健康问题。 ${ }^{[8]}$ 承担国 际航班飞行任务的乘务员, 飞行时间有时甚至会跨越多 达 8、9 个时区, 长途飞行以及机身振动、重力等因素 都会加剧空乘人员的疲劳感。从生物学角度讲, 每个生 物体都有一种叫 “生物钟” 的生理机制, 即个体生命活 动的节律性。跨时区飞行造成的时差会打破个体作息规 律, 造成空乘人员生理节律的紊乱。治疗时差反应的唯 一办法就是获得充足的休息。身体的自我调整需要一定 的恢复期，飞行时间越长、跨越的的时区越多，身体恢 复需要的时间周期也越长, 但许多空乘人员很难获得必 要的休息, 在在身体还未恢复到正常状态下又开始执行 下一班飞行任务了。为了工作需要, 很多空乘人员甚至 不得不服用神经调节类药物, 以便保证在特定的时间睡 觉或工作, 而不是依据身体的作息需要。虽然我国民航 总局对空乘人员每月飞行时数设置了最高限制, 但实际 上, 空乘人员的工作时间远不止他们实际飞行的时数, 比如飞行前必需的准备时间是不算在飞行时数内的。此 
外, 由于航空运输存在诸多不确定因素, 有时航空公司 对机组调度也不能做到完全控制, 为了最大限度降低劳 动力成本, 航空公司经常会临时调整空乘人员的飞行任 务, 这些调整包括飞行目的地的变化、飞行时间的变化、 飞机机型的变化等, 这不仅会直接导致空乘人员工作时 间和工作内容的变动, 而且意味着他们工作以外的生活 也将受到影响。 ${ }^{[9]}$ 已有统计数据表明, 空姐罹患膀胱及 尿道感染、上呼吸道感染、乳腺癌、月经失调、背部和 足部疾病、睡眠障碍及抑郁症的机率明显高于其他行业 从业者。 ${ }^{[10]}$ 除了身体上的耗竭, 空乘人员心理上也承受 着巨大压力。因为航空运输的属性决定了空乘人员必须 较长时间地离家, 而且他们的工作时间与其他职业相比 存在更多的不确定性, 这就造成了空乘人员不得不面对 工作使命与家庭责任之间的矛盾和冲突。尤其是女性空 乘人员, 由于社会性别角色的定位, 在无法承担照顾家 庭、养育子女的情况下更容易产生焦虑和自我不认同感, 导致工作效率降低、工作满意度降低等职业倦急表现。

\section{4. 特殊的工作环境与空乘人员职业倦急感}

空乘人员工作环境的特殊性体现在工作场所的高 风险性和工作对象的复杂性。虽然说飞机的安全系数是 所有交通工具中最高的, 但飞机一旦出事, 生还的希望 非常沙茫, 高空飞行依然存在着高风险的属性, 所以, 空乘人员除了服务技能外, 必须牢记和熟悉各种急救知 识和逃生方法。与地面工作相比较, 空乘工作的物理环 境具有高空高速、低压缺氧、高噪音、辐射大、低湿度、 空间封闭狭小等特点。作为普通旅客, 乘坐飞机短短几 个小时都会经常出现身体上的不适, 而且民航对于一些 有疾病的乘客或孕妇等人群乘坐飞机也作了明确的限 制和规定。因此, 可以想象这些因素给常年飞行的空乘 人员带来的健康危害。简单举例说明, 飞行过程中气压 的变化会增加中耳腔膜的内压力, 引起耳痛、耳鸣等不 适, 甚至造成耳膜穿孔和听力损失。缺氧和噪音的环境 使人体血液中胆固醇增高, 容易诱发心脑血管等方面的 问题。飞行高度的增加会加大辐射水平, 导致空乘人员 内分泌系统紊乱和激素水平异常, 低湿度、负离子缺乏 的空气也会加快皮肤衰老的速度。空乘服务工作需频繁 地低头、弯腰且以站立居多, 加大了他们罹患静脉曲张、 颈椎、腰椎等方面疾病的风险。除此之外, 在一架移动 的飞机上工作使空乘人员面临受伤的高风险。搬运沉重 的行李、头顶的行李架、服务推车也会增加工作中受伤 的风险, ${ }^{[11]}$ 由于气流颠䇦、飞机震动等因素, 极易发生 扭伤、跌倒、粱伤、被跌落的行李砸伤等意外伤害。长 期的体力劳累和病患使空乘人员无法精力充沛地投入 工作。

空乘人员的工作对象是形形色色的乘客, 无论是年 龄和性别, 还是职业、收入、国籍、种族等特征, 个体 之间都存在着巨大差异, 因此乘客的心理需求和行为表 现也大相径庭。空乘和乘客, 作为服务的提供方和受用 方, 二者之间的矛盾似乎不可避免, 其中既有非乘务员 主观原因如飞机延误、无法按时起飞等问题引起的, 也
有因信息沟通不畅等其他原因导致旅客对空乘人员的 服务不满。航空公司对空乘人员进行职业素养培训时, 都会强调 “以客为尊” 的服务理念, 要求空乘人员作为 航空公司整体形象的代言人, 当与顾客发生矛盾时, 首 先要从顾客的感受出发, 敏锐感知旅客的不满情绪, 开 展及时到位的弥补和安抚, 任何时候都要维护公司的声 誉和利益。事实上, 在空中飞行这个特殊的环境中, 民 航业提供的首要服务是安全, 空乘人员所能提供的服务 是有限的。结果双方也经常因 “安全” 问题发生矛盾, 比如 “系好安全带、打开遮光板、关闭手机、在飞机滑 行、爬升、下降阶段禁止使用卫生间, 任何松散物品不 得放在应急出口及其座位处” 等这些保证飞行安全的要 求往往也会造成某些旅客对空乘人员的不满, 甚至投诉。 航空公司相关部门在处理这类投诉时往往不考虑现实 情况, 片面地归责为乘务员的沟通能力不足。当空乘人 员在工作中的正当权益得不到保障、甚至感受不到尊重 时, 工作的积极性就会受到严重挫伤。

\section{5. 组织因素与空乘人员职业倦急感}

对于任何一个企业或单位来说, 人力资源都是最核 心的要素。个体参与组织是为了实现个人发展的需要, 而组织成员的个体行为也会直接影响组织的绩效。美国 著名管理心理学家施恩(E.H.Schein)提出了心理契约这 个概念, 揭示了组织和个体关系的本质。心理契约即“个 人将有所奉献与组织欲望有所获得之间, 以及组织将针 对个人期望收获而有所提供的一种配合。”这一无形的、 心理上契约是连接个体与组织的纽带, 我们可以把它理 解为一个组织的成长与成员的发展的满足条件在心理 上的契合, 是存在于组织和成员间的动态的心理期望。 这些存在于员工心中的期望, 我们可以用工作满意度、 归属感与安全感、职业发展空间、组织认同感等维度来 衡量。组织一旦违背员工的心理契约, 员工就很容易出 现职业倦急等问题。

拿航空公司来说, 航司内部的晋升制度、激励机制、 工作氛围、企业文化等组织因素都会直接影响空乘人员 的工作状态。在现行航司运行体制下, 空乘人员的职业 生涯大体分为初始乘务员学员、普通舱乘务员、两舱 (头 等舱、商务舱）乘务员、区域乘务长、主任乘务长这几 个阶段。尽管航空公司每年都会对乘务员进行安全、服 务技能及语言等方面的培训和考核, 并对乘务员从飞行 年限、工作绩效、工作表现等多方面进行考核, 但公司 对空乘人员职业发展的需求考虑较少, 空乘人员面临职 业发展空间狭小、晋升渠道单一的困境。另外, 航空公 司管理岗位很有限, 且晋升机制较为行政化, 当空乘人 员升至主任乘务长岗位时, 其职业生涯基本就止步了, 所以许多空乘专业学生就业以后感慨空乘是一个一眼 就能望到头的工作, 因此缺乏为之奋斗的动力, 自然工 作热情下降, 逐渐出现职业倦急感。

员工的工作绩效是员工能力和动力的双重体现, 有 效的激励机制能充分调动员工的工作积极性, 提高工作 效率, 从而使员工获得更高的工作成就感, 提升工作满 
Janssen, P.M., Vander Hulst, R., Brouwer, J. (2000) Using equity theory to examine the difference between burnout and depression, Anxiety, Stress and Coping, 13:247-268.

[5] Maslach C., Leiter, M.P. (1997) The truth about burnout: How organizations cause personal stress and what to do about it, San Francisco: Jossey-Bass:8.

[6] Brock, B.L., Grady, M.L.(2000) Rekindling the flame: Principals combating teacher burnout, Thousand Oaks, CA: Corwin Press:30.

[7] Zhuang Feifei(2018)Research on the Loss of Employees in Air China[D]:26-29.

[8] Rberta Lessor(1985) Consciousness of time and time for the development of consciousness: health awareness among women flight attendants, Sociology of Health \& Illness, Volume7,Issue 2:200.

[9] Vivian Shalla(2008)Time warped: the flexibilization and maximization of flight attendant working time, Canadian Review of Sociology, Volume 41, Issue $3: 361$.

[10] Rberta Lessor(1985) Consciousness of time and time for the development of consciousness: health awareness among women flight attendants, Sociology of Health \& Illness, Volume7,Issue 2:204.

[11] Suneth B. Agampodi, Samath D. Dharmaratne, Thilini C Agampodi(2009)Incidence and predictors of onboard injuries among Sri Lanka flight attendants, BMC Public Health 9:227.

\section{REFERENCES}

[1] Freudenberger, H. J. (1975) The staff burnout syndrome in alternative institutions, Psychotherapy Theory, 12:72-73.

[2] Maslach, C. (1978) The client role in staff burnout, Journal of Social Issues, 34(4): 111-124.

[3] Maslach, C., Jackson, S. E. (1981) The measurement of experience burnout, Journal of Occupational and Behavioral Science, 2:99-113.

[4] Bakker, A.B., Schaufeli, W.B., Demerouti, E., 